\title{
Transport of Intensity Equation applied to Study Quasi-Dynamic Surface and Boundary Reconstruction
}

\author{
Kazuo Ishizuka
}

HREM Research Inc, Matsukazedai, Saitama 355-0055, Japan

The surface and boundary is very important to control materials property, and electron microscopy is one of the most powerful techniques to study their atomic structure. However, studying such a non-periodic structure is hampered by aberrations, especially spherical aberration, of an objective lens, even when we use a modern high-resolution transmission electron microscope (HRTEM). This is one of the motivations to develop a Cs (spherical aberration) corrector. Recently, a Cs-corrected HRTEM becomes commercially available. However, many samples in electron microscopy are phase objects as in the case of optical microscopy. Since it is difficult to realize an analogue of the Zernike phase plate for electrons, a simple technique proposed by Scherzer [1] to observe a phase object by adjusting the defocus is till used even with a Cs-corrected microscope. In this case an information transfer is still determined by the phase transfer contrast function. Thus, low frequency components are less transferred than the high frequency components, and it is difficult to obtain a quantitative result from a single image obtained at the Scherzer defocus.

On the other hand there are working algorithms to reconstruct a wave front (complex wave function) from a series of through-focus images. The maximum likelihood method [2] and the Wiener filter method [3] usually use about twenty images, while the Gerchberg-Saxton type iteration method [4] requires normally five images. After reconstructing a wave front, we may be able to correct any aberrations including spherical aberration, and use information beyond the Scherzer limit [1]. Moreover, all of these techniques use a series of images taken at a wide defocus range, and thus recover also low frequency components which will not be recorded in the image taken at the Scherzer defocus.

We have demonstrated recently that the technique based on the Transport of Intensity Equation (TIE) proposed by Teague [5] can be applied to evaluate a phase distribution even at atomic resolution [6]. The TIE is an equation for wave propagation in terms of a phase and intensity under the small angle (paraxial) approximation as described below:

$$
\frac{2 \pi}{\lambda} \frac{\partial}{\partial z} I(x y z)=-\nabla_{x y} \cdot\left(I(x y z) \nabla_{x y} \phi(x y z)\right)
$$

where $\nabla_{x y}$ is a derivative operator working in the $(\mathrm{x}, \mathrm{y})$ pane. This equation says that a phase distribution at a specific plane may be estimated from only an intensity distribution at the same plane and its derivative along the wave propagation direction. Therefore, we don't need intensity information over a wide range of defocus as required by other wave reconstruction algorithms [2-4]. However, in order to evaluate the intensity derivative numerically, we have to measure intensities at sufficiently close image planes. Although an accurate estimate of the intensity derivative may be given by a difference of intensities measured at sufficiently close image planes, the intensity difference may not be significant with respect to noise in the images. Conversely, the intensity difference with a large defocus step will give a poor estimate of the intensity derivative. For the symmetric three-image case, an intensity difference defined by

$$
\frac{\Delta \Psi}{\Delta z} \equiv \frac{\Psi(x y, z+\varepsilon)-\Psi(x y, z-\varepsilon)}{2 \varepsilon}
$$

may be a good approximation for the derivative when the following condition is satisfied [6]:

$$
\left(\pi \lambda \varepsilon g_{\max }^{2}\right)^{2} / 3 ! \leq c<<1
$$


where $g_{\max }$ is the highest special frequency to be recovered, and $\mathrm{c}$ is a small number, say 0.25 . Then, the upper defocus limit $(2 \varepsilon)$ for a resolution of $0.14 \mathrm{~nm}$ assuming $400 \mathrm{kV}$ electrons is $9.5 \mathrm{~nm}$, which will give a significant intensity change even of high-resolution images.

Since we need only three images to estimate the phase distribution, we can estimate a series of phase distributions using a set of through-focus images. Here, we use a set of twenty-five images of Au obtained with a focus step of $2.4 \mathrm{~nm}$ using a Philips CM300 at NCEM equipped with a field emission gun. Phase distributions are calculated by using the software, QPt for DigitalMicrograph [7], which was developed based on the algorithm proposed by Paganin and Nugent [8] to solve the TIE. Figure 1 shows two phase distributions obtained from a first part of the data set, namely 1, 3 and 5, and a middle part, namely 12, 14 and 16 . Here, we back-propagate the wave to the specimen exit surface assuming a nominal defocus value. Therefore, the difference between the two phase distributions at the surface as well as the interface will reflect structure change between the exposure times. Thus, this technique will open a possibility to investigate a quasi-dynamic process during an order of second.

\section{Refedrences}

[1] O. Schrzer, J. Appl. Phys. 20 (1949) 20-29.

[2] W.M.J. Coene, A. Thust, M. Op de Beek, D. Van Dyck, Ultramicroscopy 64 (1996) 109-135.

[3] R.R. Meyer, A.I. Kirkland, WO. Saxton, Ultramicroscopy 92 (2002) 89-109.

[4] L.J. Allen, W. McBride, N.L. O’Leary, M.P. Oxley, Ultramicroscopy 100 (2004) 91-104.

[5] M.R. Teague, J. Opt. Soc. Am. 73 (1983) 1434-1441

[6] K. Ishizuka, B. Allman, J. Electron Micros. (2005) submitted.

[7] A TIE plug-in for DigitalMicrograph (Gatan Inc), see www.hremresearch.com for details.

[8] D. Paganin, K.A. Nugent, Phys. Rev. Lett. 80 (1998) 2586-2589.

[9] The author greatly acknowledges Dr. Christian Kisielowski for providing the Au data.
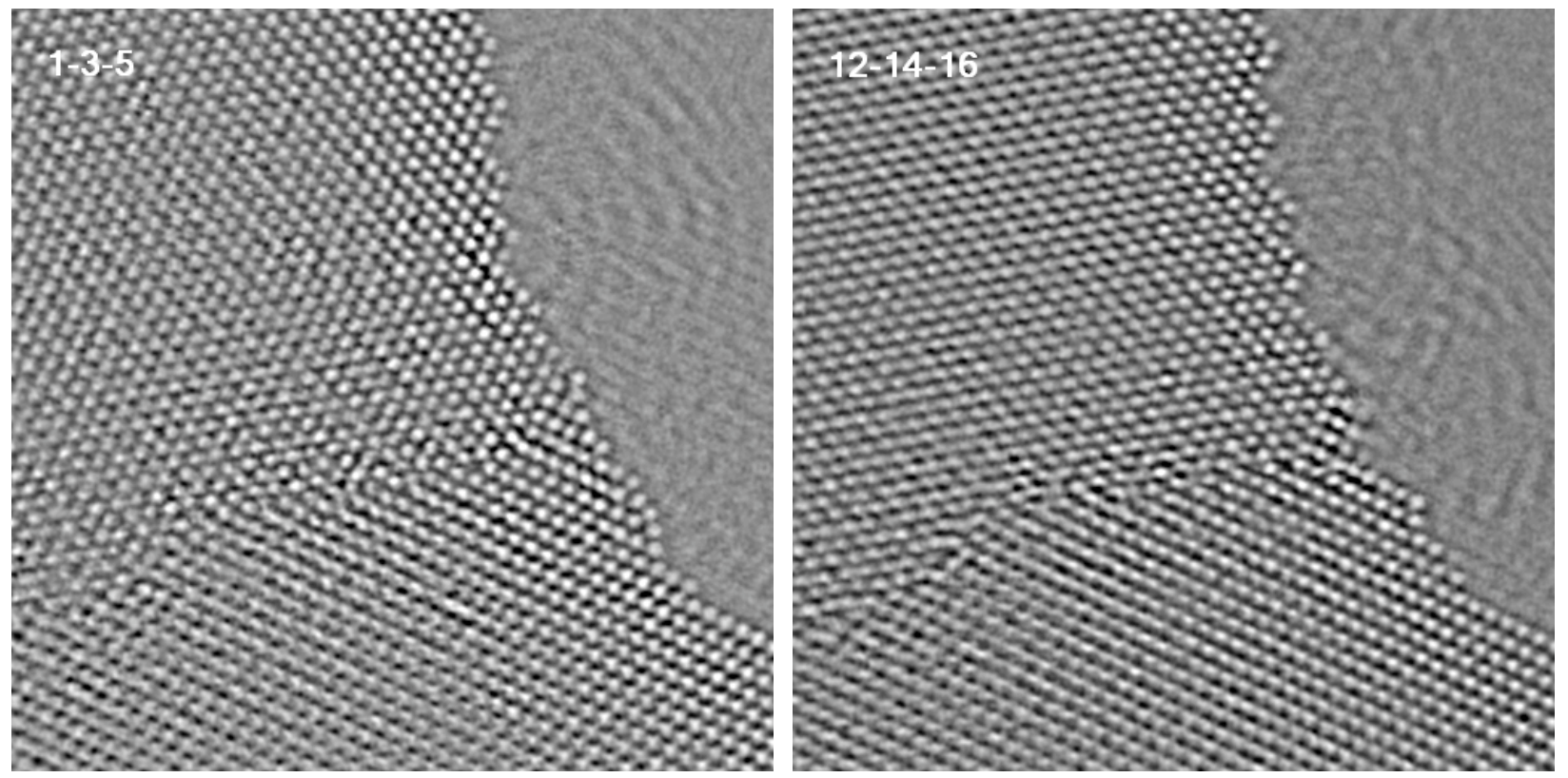

Figure 1. Two phase distributions obtained from three images of a first part and a middle part of the data set of twenty-five Au images. Difference of the two phase distribution at the surface as well as the interface will reflect structure change between the exposure times. 